\title{
DWDM Fiber-Wireless Access System with Centralized Optical Frequency Comb-based RF Carrier Generation
}

Pang, Xiaodan; Beltrán, Marta; Sánchez, José; Pellicer, Eloy; Vegas Olmos, Juan José; Llorente, Roberto; Tafur Monroy, Idelfonso

Published in:

2013 Optical Fiber Communication Conference and Exposition and the National Fiber Optic Engineers Conference (OFC/NFOEC)

Link to article, DOI:

10.1364/NFOEC.2013.JTh2A.56

Publication date:

2013

Link back to DTU Orbit

Citation (APA):

Pang, X., Beltrán, M., Sánchez, J., Pellicer, E., Vegas Olmos, J. J., Llorente, R., \& Tafur Monroy, I. (2013). DWDM Fiber-Wireless Access System with Centralized Optical Frequency Comb-based RF Carrier Generation. In 2013 Optical Fiber Communication Conference and Exposition and the National Fiber Optic Engineers Conference (OFC/NFOEC) (pp. JTh2A.56). IEEE. https://doi.org/10.1364/NFOEC.2013.JTh2A.56

\section{General rights}

Copyright and moral rights for the publications made accessible in the public portal are retained by the authors and/or other copyright owners and it is a condition of accessing publications that users recognise and abide by the legal requirements associated with these rights.

- Users may download and print one copy of any publication from the public portal for the purpose of private study or research.

- You may not further distribute the material or use it for any profit-making activity or commercial gain

- You may freely distribute the URL identifying the publication in the public portal 


\title{
DWDM Fiber-Wireless Access System with Centralized Optical Frequency Comb-based RF Carrier Generation
}

\author{
Xiaodan Pang ${ }^{1}$, Marta Beltrán ${ }^{2}$, José Sánchez ${ }^{2}$, Eloy Pellicer ${ }^{2}$, J.J. Vegas Olmos ${ }^{1}$, Roberto Llorente ${ }^{2}$, \\ and Idelfonso Tafur Monroy ${ }^{1}$ \\ 1) DTU Fotonik, Technical University of Denmark, Oersteds Plads 358, 2800 Kgs. Lyngby, Denmark \\ xipa@fotonik.dtu.dk \\ 2) Valencia Nanophotonics Technology Center, Universidad Politécnica de Valencia, 46022 Valencia, Spain
}

\begin{abstract}
We propose and experimentally demonstrate an optical wireless DWDM system at $60 \mathrm{GHz}$ with optical incoherent heterodyne up-conversion using an optical frequency comb. Multiple users with wireline and wireless services are simultaneously supported. OCIS codes: (060.2330) Fiber optics communications; (060.5625) Radio frequency photonics; (060.2840) Heterodyne
\end{abstract}

\section{Introduction}

Wireless communication is an area that has witnessed substantial technology advancement in the past two decades. End-users have benefited from such advances and a large fragment of our economy is growing around broadband communication services, such as social networking, cloud computing, e-health systems, video streaming, gaming and so on. Capacity improvements in both optical networks and wireless systems have been based on incremental refinements of existing technologies. However, it is becoming evident that this growth model may not provide the projected future capacity - conservative estimations project a 18-fold increase between 2011 and 2016 in global mobile data traffic. Furthermore, the average smartphone will generate 2.6 GB of traffic per month in 2016, a 17fold increase over the 2011 average of $150 \mathrm{MB}$ per month. Aggregate smartphone traffic in 2016 will be 50 times greater than it is today, with a compounded annual growth rate of 119 percent [1]. Current technologies will not support such traffic growth, since wireless bands are already saturated. Millimeter-wave at $60 \mathrm{GHz}$ band is viewed as a promising candidate with its $7 \mathrm{GHz}$ spectrum available for radio communication $(57-64 \mathrm{GHz})[2,3]$.

On the other hand, seamless convergence between fiber-optic and wireless networks in the "last mile" has a great potential for delivering data services to the end-users with more flexibility and mobility [4]. In optical access networks, wavelength division multiplexing (WDM) technique is considered as a promising candidate as it can increase the total throughput as well as ensure the scalability of the network by allocating wavelengths to each end user $[5,6]$. However, when integrating the conventional radio-over-fiber (RoF) signals at $60 \mathrm{GHz}$ and above with the dense WDM (DWDM) system with $50 \mathrm{GHz}$ or $25 \mathrm{GHz}$ channel spacing, the signal carriers will be filtered out by the arrayed waveguide gratings (AWG). Meanwhile, the fiber chromatic dispersion induced double-side band RoF signal power fading will further limit the flexibility of the system. In this paper, we propose an optical fiberwireless DWDM access system using optical incoherent heterodyne up-conversion by employing an centralized optical frequency comb (OFC)-based local oscillator (LO). As the carrier signals are added after the AWG, no adaptation to the baseband DWDM signals is needed and the system flexibility is preserved. The proposed system has the potential to simultaneously support multiple users with both wireline and wireless broadband services.

\section{Architecture of the optical wireless DWDM network}
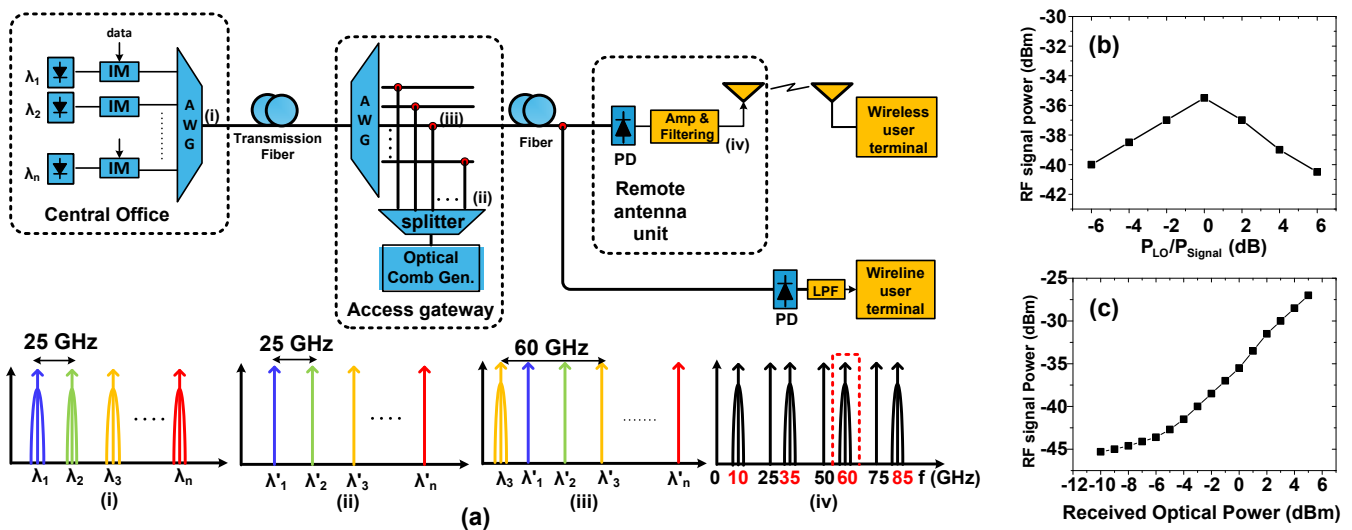

Figure 1. (a). Conceptual diagram of the proposed optical wireless DWDM system using optical frequency comb-based incoherent heterodyne up-conversion. (b). Measured $60 \mathrm{GHz}$ RF power vs. ratio of LO power and signal power. (c). RF power vs. optical power when $\mathrm{P}_{\mathrm{LO}} / \mathrm{P}_{\text {signal }}=0 \mathrm{~dB}$ 
Figure 1 shows the conceptual diagram of the proposed optical fiber-wireless DWDM access system. In the central office, multiple CW lightwaves from $\lambda_{1}$ to $\lambda_{n}$ separated by $25 \mathrm{GHz}$ are used to carry the baseband data (Inset (i) in Fig. 1 (a)). An AWG aggregates the signals into a transmission fiber. A second AWG at the access gateway is used to separate the wavelengths that are assigned to specific users. An optical frequency comb from $\lambda_{1}{ }^{\prime}$ to $\lambda_{n}{ }^{\prime}$ with $25 \mathrm{GHz}$ frequency separation is employed as a local oscillator (LO) and combined with each incoming signal. Since the shift between $\lambda$ and $\lambda^{\prime}$ is $10 \mathrm{GHz}$, we observe a $60 \mathrm{GHz}$ separation between the corresponding comb line and the signal, shown in Inset (ii) and (iii) in Fig. 1. The combined signals are then sent to the wireline/wireless end-users. For the wireline user, the baseband signal is directly detected by a low frequency PD followed by a low-pass filter (LPF). For wireless applications, the signal is sent to the remote antenna unit (RAU), where the heterodyne mixing is performed at a fast-response PD. As shown in Inset (iv) in Fig. 1, the baseband signal is simultaneously upconverted to different RF bands including X-band, $\mathrm{K}_{\mathrm{a}}$-band, V-band and W-band. Signal amplification and filtering are performed to select the on-demand RF band based on the user applications.

An initial characterization of the generated RF signal at $60 \mathrm{GHz}$ is firstly carried out. We use two free-running $\mathrm{CW}$ lasers with $<100 \mathrm{kHz}$ linewidth to perform the incoherent heterodyning mixing. Fig. 1 (b) shows the generated RF power as a function of the power ratio between the two lasers. The optimal power ratio between the two lasers is found at $0 \mathrm{~dB}$, meaning that when the two lasers gives equal power, the RF signal is maximized. This result is consistent with our previous theoretical analysis [7]. The RF power with respect to the combined optical power is shown in Fig. 1 (c), where the lasers power ratio is optimized.

\section{Experimental setup and results}

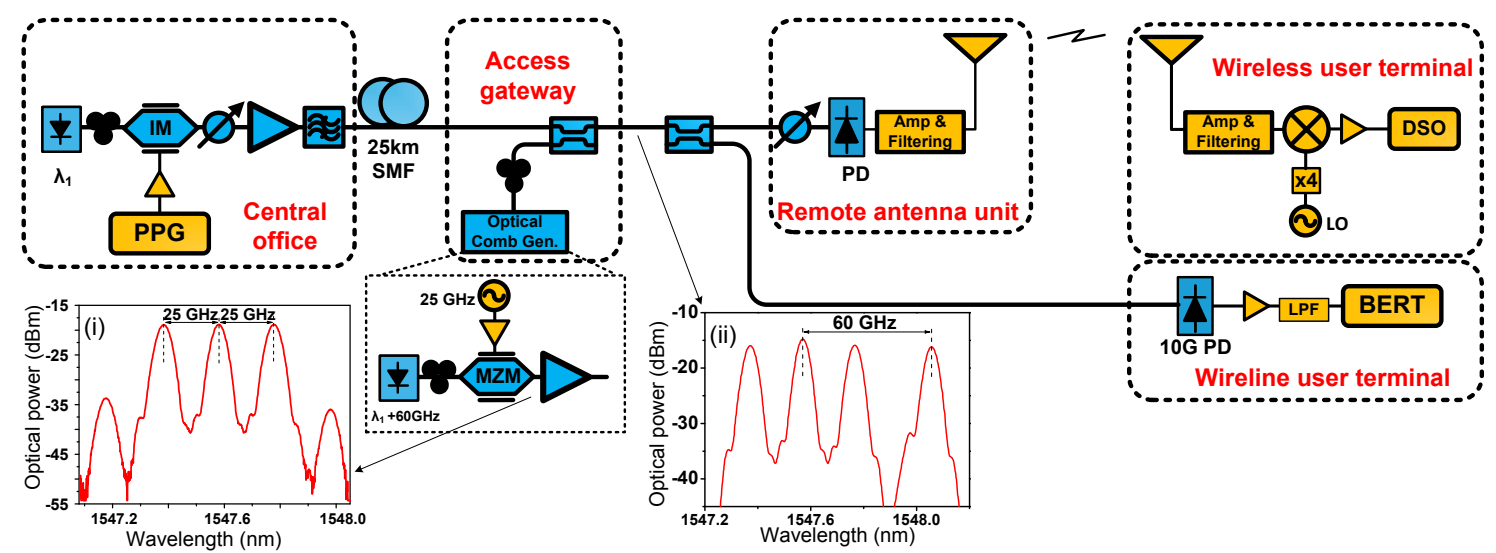

Figure 2. Experimental setup. PPG: pulse-pattern generator; BERT: bit-error-ratio tester; DSO: digital storage oscilloscope; LPF: low-pass filter.

Figure 2 shows the experimental setup for a single optical channel. A CW lightwave emitted from a Distributed Feedback Laser (DFB, $\lambda_{1}=1548.05 \mathrm{~nm}$ ) with $<100 \mathrm{kHz}$ linewidth is modulated via an intensity modulator (IM) driven by a $2.5 \mathrm{~Gb} / \mathrm{s}$ pseudo-random binary sequence (PRBS) electrical signal of length $2^{7}-1$ to generate an On/OFF keying (OOK) optical baseband signal. An erbium-doped fiber amplifier (EDFA) is employed for amplification, and an optical bandpass filter (OBPF) with $0.8 \mathrm{~nm}$ bandwidth is used to filter the out-of-band noise. After that, the signal is transmitted to the access gateway via a $25 \mathrm{~km}$ standard single mode fiber (SSMF) link. A second DFB laser $\left(\lambda_{2}=1547.57 \mathrm{~nm}\right)$ with $<100 \mathrm{kHz}$ linewidth is launched into an OFC generator employing an overdriven MachZehnder modulator (MZM). The MZM is biased in its nonlinear region by equalizing the power of the central 3 comb lines that are separated by $25 \mathrm{GHz}$ (Fig. 2. (i)). After amplification the OFC is combined with the baseband signal at a $3 \mathrm{~dB}$ coupler. The separation between the signal and the center comb line is $60 \mathrm{GHz}$, as shown in Fig. 2. (ii). The signal is then split into two paths, one going directly to the wireline user terminal where a low frequency PD performs $\mathrm{O} / \mathrm{E}$ conversion of the baseband signal before sending it to the bit-error-ratio tester (BERT) for BER evaluation; the other transmitting to the RAU where the signals heterodyne mixing takes place at a $60 \mathrm{GHz}$ PD. Following photodetection and amplification, a RF filter with $3 \mathrm{~dB}$ bandwidth ranging from $56.26-62 \mathrm{GHz}$ is used to select the up-converted signal centered at $60 \mathrm{GHz}$. The filtered RF signal then feeds a standard V-band horn antenna of $20 \mathrm{dBi}$ for up to $6 \mathrm{~m}$ wireless transmission. An identical horn antenna is used to pick up the signal at the receiver.

In order to detect the RF signal transparently to modulation formats, an electrical mixer is used to perform the down-conversion. A $14 \mathrm{GHz}$ signal generator and a frequency multiplier by 4 are employed to generate the LO signal for down-conversion. The transmitted RF signal centered at $60 \mathrm{GHz}$ is then converted to an intermediate frequency (IF) at $4 \mathrm{GHz}$. The analog to digital conversion (A/D) is realized in a digital storage scope with $40 \mathrm{GS} / \mathrm{s}$ 


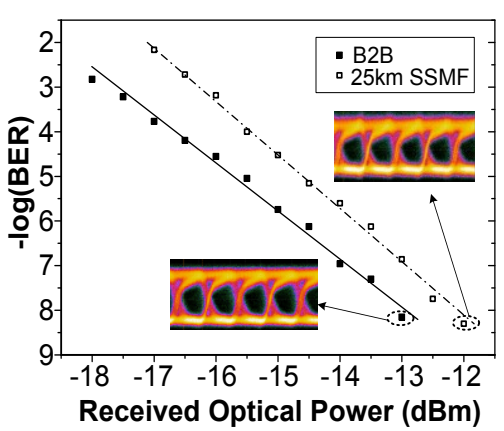

(a)

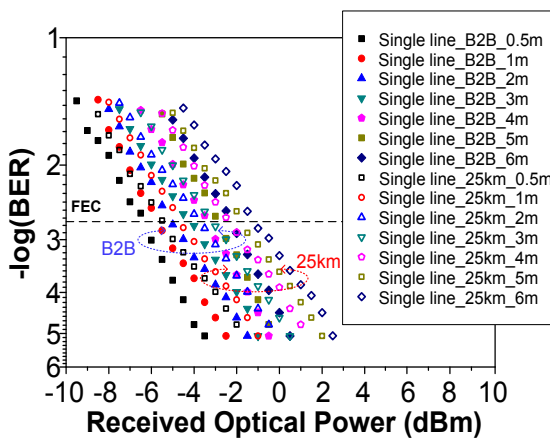

(b)

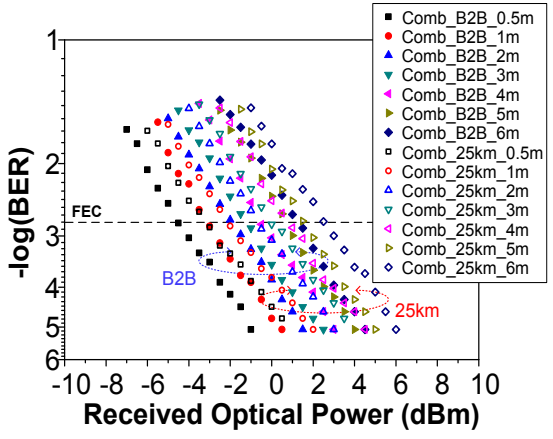

(c)

Figure 3. BER curves for (a) baseband signal; (b) the $60 \mathrm{GHz}$ wireless signal in the case of single line LO; (c) the $60 \mathrm{GHz}$ wireless signal in the case of optical frequency comb-based LO

and $13 \mathrm{GHz}$ bandwidth. The signal demodulation is performed by offline digital signal processing (DSP). To overcome the frequency jitter that usually occurs with optical incoherent heterodyne mixing, a digital selfheterodyning is firstly performed to further down-convert the IF signal to baseband in the digital domain. The signal then passes a LPF followed by the decision and BER evaluation.

First, the performance of the baseband signal received by the wireline user terminal is evaluated. Figure 3 (a) shows the BER curve of the baseband signal before and after the $25 \mathrm{~km} \mathrm{SSMF} \mathrm{link.} \mathrm{Negligible} \mathrm{shape} \mathrm{distortion} \mathrm{of}$ the received eye diagram is seen after the fiber transmission. A power penalty of less than $1 \mathrm{~dB}$ is observed coming from the fiber dispersion. We then evaluate the wireless transmission performance. To begin with, we firstly assess the case that the LO is a single optical line instead of the OFC by switching off the $25 \mathrm{GHz}$ generator. The BER curves of different wireless distances from $0.5 \mathrm{~m}$ till up to $6 \mathrm{~m}$ for both B2B and after $25 \mathrm{~km}$ SSMF are shown in Fig. 3 (b). We can see that for all cases the BER performance yield values below the FEC limit of $2 \times 10^{-3}$ without an apparent BER floor. Compared with B2B, the penalty after $25 \mathrm{~km}$ SSMF transmission at the FEC limit is around $2 \mathrm{~dB}$ for all wireless distances. After this, we substitute the LO with the OFC generator, while keeping the peak power of each comb line the same level with the single line case. Figure 3 (c) shows the BER performance of the cases that OFC-based LO is employed for signal up-conversion. Similarly, for all cases with and without $25 \mathrm{~km}$ SSMF transmission plus up to 6 meters wireless transmission, BERs of well below the FEC limit are achieved. Compared with the single-line $\mathrm{LO}$ case, we observe around $3.5 \mathrm{~dB}$ difference in performance in terms of sensitivity at the FEC limit. This difference is from the power of the adjacent comb lines, which are filtered out before the wireless transmission. For the case of $25 \mathrm{~km}$ SSMF plus 6 meters wireless transmission, the requirement for the received optical power at the BER of $2 \times 10^{-3}$ is $2.5 \mathrm{dBm}$.

\section{Conclusion}

We have proposed an optical fiber-wireless DWDM access system that simultaneously provides wireline and wireless broadband services with no adaptation to the baseband signals while preserving the system flexibility. The $60 \mathrm{GHz} \mathrm{mm}$-wave is generated using the optical incoherent heterodyne up-conversion method with an optical frequency comb-based LO. A $60 \mathrm{GHz}$ signal has been experimentally generated with a LO of 3 comb lines with $25 \mathrm{GHz}$ separation mixing with a $2.5 \mathrm{Gbit} / \mathrm{s}$ OOK optical baseband signal. Signal transmission through a $25 \mathrm{~km}$ SSMF and up to 6 meters air distances is successfully received with a BER performance well below $2 \times 10^{-3}$. The optical power penalty for the $60 \mathrm{GHz}$ mm-wave signal after SMF transmission is around $2 \mathrm{~dB}$.

\section{References}

[1] "Cisco Visual Networking Index: Forecast and Methodology, 2011-2016," Cisco White Paper, May, 2012, [Available: Online]

[2] J. Yu et al., "A Novel Architecture to Provide Super-Broadband Optical Wireless Access Service," OFC/NFOEC 2012, paper JTh2A.70.

[3] W.J. Jiang et al., "40 Gb/s RoF Signal Transmission with $10 \mathrm{~m}$ Wireless Distance at $60 \mathrm{GHz}$," OFC/NFOEC 2012, paper OTu2H.1.

[4] D. Zibar et al., "Hybrid Optical Fibre-Wireless links at the 75-110 GHz Band Supporting 100 Gbps Transmission Capacities," MWP 2011, paper 3005.

[5] T. Tashiro et al., "40 km fiber transmission of time domain multiplexed MIMO RF signals for RoF-DAS over WDM-PON," OFC/NFOEC 2012, paper OTu2H.4.

[6] K. Prince et al., "Converged Wireline and Wireless Access Over a 78-km Deployed Fiber Long-Reach WDM PON," IEEE PTL, 21, pp. 12741276, (2009).

[7] X. Pang et al., "25 Gbit/s QPSK Hybrid Fiber-Wireless Transmission in the W-Band (75-110 GHz) With Remote Antenna Unit for InBuilding Wireless Networks," Photonics Journal, IEEE, 4, pp. 691-698, (2012) 